\title{
Opportunities and Challenges of Robotics and Automation in Offshore Oil \& Gas Industry
}

\author{
Heping Chen"1, Samuel Stavinoha', Michael Walker', Biao Zhang'², Thomas Fuhlbrigge ${ }^{2}$ \\ ${ }^{1}$ Ingram School of Engineering, Texas State University, San Marcos, USA \\ ${ }^{2}$ ABB Corporate Research Center, Windsor, USA \\ Email: Heping.chen@txstate.edu, Biao.zhang@us.abb.com
}

Received 21 May 2014; revised 25 June 2014; accepted 10 July 2014

Copyright (C) 2014 by authors and Scientific Research Publishing Inc.

This work is licensed under the Creative Commons Attribution International License (CC BY).

http://creativecommons.org/licenses/by/4.0/

C. (i) Open Access

\section{Abstract}

The oil and gas industry will continue to boom in the coming few decades. Obtaining oil and gas from conventional and non-conventional resources will become more and more challenging. This intensifying need will impose very considerable demands on work force, financial and technology capabilities. Since the future supplies of oil and gas are to expand, advanced technology will become increasingly necessary to obtain access to more challenging conventional and non-conventional resources. Therefore oil and gas technologies will be very costly to operate in the coming future due to hostile, hard-to-reach environments. The offshore oil industry will become a complicated myriad of advanced equipment, structures, and work force. Our objectives are to identify potential applications and research directions of robotics and automation in the oil $\&$ gas field and explore the obstacles and challenges of robotic and automation applications to this area. This study performs the necessary survey and investigation about the work conditions of robotics and automation equipment in the oil and gas industry, especially offshore oil rigs. The oil \& gas industry processes are first investigated. The personals and tasks are then explored. Furthermore, this paper reviews the current robotic automation technology. The challenges and requirements are identified for robotics and automation equipment in the oil and gas industry. The requirements of robotics and automation in the oil \& gas industry are presented. Future research opportunities are discussed from a technical perspective.

\section{Keywords}

Oil \& Gas, Robot, Automation, Hazardous Environment, Teleoperation, Offshore Oil \& Gas Industry 


\section{Introduction}

The oil and gas industry will continue to boom in the coming few decades. The oil and gas demand will grow rapidly in the next two decades [1].

The intensifying need to obtain oil and gas from more hostile, hard-to-reach environments will increase the operation cost rapidly in the coming future. Hence, the oil and gas industry keeps looking for lower-cost solutions.

To be competitive and to improve their profit margins, oil \& gas companies are committed to cost reduction. They also look for ways to minimize employee costs and improve manufacturing efficiencies and quality besides seeking lower-cost suppliers and less-expensive raw materials. Because of the rising cost of employee salary and benefits like health care, the cost reduction effort in oil \& gas companies is offset. Also high employee turnover adds the costs of retraining. Therefore, the oil and gas companies are looking for new technologies to reduce the labor cost. Also safety is a big concern in the oil and gas production. Using robotics in inspection, maintenance and repair could greatly improve the safety and efficiency.

The oil \& gas industry's presence is evident in its global networks of market supply and demand relationships. When there are fluctuations, regardless of their origins, consumers are affected in all over the world. Prices respond to changing markets with upward volatility because of an inelastic demand for oil and petroleum products. The period of high oil prices from 2004 through 2008 led to a steady demand for petroleum. As this market trend persists, oil \& gas companies have a window of opportunity to maximize efficiency and productivity to moderate the petroleum market.

One solution to both the need for efficiency and maximum production and the capabilities required to further exploration is to implement robotics and automation in offshore oil \& gas environments. Because the offshore oil \& gas processes require advanced technologies, offshore environments will deploy the safest, most secure and consistent operations by utilizing industrial robotics and automation, and the latest software and mechanical devices.

In order to investigate the challenges of robotics and automation in oil and gas industry, the necessary survey and investigation about the oil \& gas industry processes, the personals and tasks should be explored first. The work conditions must be discussed to explore the requirements of robotics and automation equipment in the oil and gas industry, especially on offshore rigs. To meet the requirements and develop robotics and automation equipment in such work conditions, this paper reviews the current technology that has been developed and discusses the future research opportunities in the oil and gas industry.

\section{Oil and Gas Industrial Processes}

The offshore oil \& gas industry is a complicated myriad of advanced equipment, structures, and work force. With a proper knowledge of offshore oil and gas rig environments, the applications of industrial robotics and automation are less abstract. Before any real vision of the potential roles robotics and automation in offshore oil processes can emerge, those processes must be enumerated appropriately.

There are many products and services related to oil and gas with an equally substantial potential for markets within the industry. There are three stages through which petroleum products pass: upstream, downstream and midstream models.

The upstream oil sector commonly refers to the searching for, recovery and production of crude oil and natural gas. It is also known as the exploration and production ( $E \&$ P) sector, including searching for potential oil and gas fields, drilling of exploratory wells, and subsequently operating the wells to recover and bring the crude oil and/or natural gas to the surface [2].

The midstream oil and gas sector is the relay point for the upstream sector's products. Midstream processes commonly refer to processing, transport, and storage of these products [3]. Because it is possible to produce pipeline quality gas for direct sale to an interstate or intrastate natural gas pipeline in the midstream sector, some treatment or processing of natural gas may occur in the midstream sector and bypass the downstream oil and gas sector completely. The midstream typically links the supply of the oil industry to the demand for energy commodities [3].

The downstream oil sector refers to the refining of crude oil and the selling and distribution of natural gas, as well as other products derived from crude oil such as liquefied petroleum gas (LPG), gasoline or petrol, jet fuel, diesel oil, other fuel oils, asphalt and petroleum coke [4] [5]. The downstream industry touches consumers 
through thousands of products. These products include petrol, diesel, jet fuel, heating oil, natural gas and propane to asphalt, lubricants, synthetic rubber, plastics, fertilizers, antifreeze, pesticides, and pharmaceuticals [4] [5].

This paper provides a fundamental knowledge base to utilize in understanding the environment in which offshore industrial robotics and automation would operate, as well as cover the challenges that exist within that environment. The processes described here relate primarily to the upstream oil and gas sector, as that particular sector hosts the majority of opportunities for robotics and automation. The oil and gas processes and the three sector model they fall into tend to parallel across the onshore and offshore industries, however the processes will be distinguished when necessary if a distinct observation is being made.

The major oil and gas extraction processes include the materials and equipment used and the processes employed. There are four major processes in the oil and gas extraction industry: (1) exploration, (2) well development, (3) production, and (4) site abandonment [6]. After these processes are completed, the production process enters. It is likely the process in which robotics and automation have the largest potential to increase efficiency and create a safer environment for offshore oil and gas rigs, all while cutting construction costs for human necessitated rig designs [6].

After the Deep Water Horizon oil spill in 2010, the Bureau of Ocean Energy Management, Regulation and Enforcement (BOEMRE) has implemented new mandatory regulations to replace old protocols for the offshore oil \& gas industry. Consequences of these new regulations are evident in the day-to-day operations in the oil \& gas industry, especially regarding inspection and maintenance of equipment. This is one area of operation that robotics and automation can dramatically improve efficiency, precision, safety, and decrease costs to companies. It is no stretch of imagination to suggest that robots and automation will soon be the primary means to effectively satisfy many of these new regulations.

\section{Challenges and Requirements}

In this section, the environments that the robotics and automation will be deployed are investigated. The hardware and software requirements are discussed.

\subsection{Challenges in Oil and Gas}

The deep waters of the Gulf of Mexico, the frigid regions of Russia, and the hot, dusty, undeveloped deserts of the Middle East are merely the geographic challenges facing today's oil and gas exploration and production industry [7]. The work conditions on offshore installations are the first thing to look at when analyzing the environments. The most important ones are as follows:

- Atmosphere: The atmospheric conditions on offshore platforms are quite unfriendly. Due to the substances used and generated during the processing of hydro carbon resources, the following three types of gases can occur separately and combined: explosive, toxic and corrosive.

- Unsheltered maritime environment: Except for the living quarters and a few technical rooms offshore platforms are partially sheltered and unsheltered. This means there is no sufficient protection against saltwater spray and direct sun light which is also reflected from the sea surface.

- Heavy weather: Wind with high speed and squalls, rain, hail and snow. All these weather conditions occur more often and more intense offshore than onshore.

- Extreme ambient temperature: Depending on the region the platform is located there can be extreme high and low temperatures. Humidity is also ranging from lower values up to condensing.

- Constraint space and/or walkways: The width of typical walkways is about $0.7-0.75 \mathrm{~m}$.

Offshore rigs have further logistical issues: (a) it is highly expensive to have people working on the rig as they must be housed and protected; (b) in the case of emergency, it must be possible to evacuate personnel quickly. As oil and gas exploration pushes into more hostile and remote regions, these difficulties become serious obstacles to the financial viability of an offshore installation.

With a basic comprehension of the processes and the personnel tasks involved in the offshore oil and gas industry, the lingering factor is the role that robotics and automated systems could potentially fill. While this is a relatively young market, there are a few examples of robotics and automation used for oil processes, but only a few. Reason for such hesitation derives from the logistical challenges that come with the implementation of a robot or an automated system in an offshore environment, as well as from a general lack of prototypes on the market. 
The disaster at the Macondo Prospect, which is called the Deepwater Horizon oil spill [8], has spawned a great deal of movement and some legislation. Much of the legislation requires an increase in inspection and maintenance at offshore rigs, where robotics and industrial automation will find a role in enabling those in the industry to meet these new standards.

To generate application scenarios for mobile robots, the operations carried out on these types of platforms must be understood. There are scheduled and occasional operations. The scheduled operations are tasks planed in the daily operation schedule. The occasional operations are those triggered by external influence on a more or less random basis. The most important scheduled operations are:

- Inspection: gauge readings and valve and lever position readings

- Monitoring: gas level, check for leakage, acoustic anomalies, surface condition and check for intruders.

- Maintenance: gas and fire detector test, sampling, pigging, cleaning, refilling and pipelines.

The most frequent occasional operations are:

- Valve and lever operation: change pressure, change flow rate and start or stop equipment operation.

- Gas leakage: identify and locate, stop dangerous operations (welding, cutting, ...) and secure area and stop leakage and monitor concentration drop.

- Fire: identify and locate fire.

The robot will have to operate at various levels of automation: fully automatic, semi-automatic and manual. Fully automatic operations require no human intervention. There will be various tasks using semi-automatic operations, which will require varying degrees of human interaction. This is quite different from the more traditional industrial robotic applications because human decision makers must be within the control loop to collaborate with the robots and the control system. The successful application of robotics and automation in the oil and gas industry will rely on the seamless integration of man, technology, and organization. Compared to the fully automated offshore robots, the inspection robots are the simplest because they may need constant human involvement. The manipulation robot is more complex than the inspection robot because it has to make decisions while performing different tasks. In the short term, the inspection robot could have many applications in the oil \& gas industry.

\subsection{Requirements for Hardware Development}

In order to be suitable for dependable and useful offshore operations, the following basic requirements must be met by the hardware of the mobile inspection and manipulation robots:

- The robot must be certified-or as a prototype be certifiable. The robotic system must be explosive-proof, weather-proof and salt-water-proof [9].

- The electronics must be suitable for harsh environments.

- The drive systems of the robot must be suitable for the hostile environments and the very special floor conditions.

- The robot has to maneuver in confined spaces. Therefore its size must be adapted to the previously defined reference passage.

- The robot must be equipped with highly reliable sensors to perceive its surroundings, especially to detect obstacles.

- If the robot is supposed to navigate in different levels, the robot must be able to move vertically on simple ladder-type steel profiles.

- The robot must be equipped with sensors to track its position for autonomous motion.

- The robot must be equipped with a manipulator to handle objects and position sensors.

- The robot must have appropriate application sensors and tools to execute inspection and manipulation tasks autonomously, semiautonomously or manually.

- The robot must be able to communicate with a central control station, for example by Wireless LAN.

\subsection{Requirements for Communication}

A major challenge for teleoperation within the oil and gas industry is the remote nature of offshore installations. The offshore rigs can be located hundreds of miles away from land, conducting complex and dynamic operations in harsh environments.

Operation failures in such installations may result in major consequences for human operators, the environment and process equipment. Safe and efficient teleoperation is critical for such unmanned facilities, securing benefit and optimal productivity at remote locations. 


\subsection{Requirements for Software Development}

A mobile robot for inspection and manipulation in offshore environments may only be acceptable if it can be used without expert knowledge but rather easily and intuitively as a daily-used tool. This implies that:

- the robot can be controlled manually, semi-automatically and autonomously;

- new inspection and manipulation tasks can be programmed quickly and without the assistance of specialists;

- anyone working next to the robot can interact with it safely.

Therefore, the control software for a mobile offshore robot should provide functions to

- navigate on the platform without collisions both in remote-controlled mode and in automatic mode, for example move to a given target autonomously;

- easily program typical inspection, monitoring and/or manipulation tasks;

- execute pre-programmed inspection, monitoring and/or manipulation tasks automatically;

- enable supervision and control of the robot from a remote location;

- display sensor data such as camera images, the current gas concentration or other sensor information on the remote screen;

- alert the remote operators when abnormal sensor values are detected;

- review sensor recordings of past autonomous inspection tasks at the remote screen.

\subsection{Requirements for Robotic Systems}

Because it is installed at remote and isolated places, offshore oil and gas platforms pose a challenging environment for their human operators due to the unsheltered maritime environment, heavy weather and unfriendly, often explosive, toxic and corrosive atmosphere. In order to apply mobile robotics technologies in offshore environments, a number of challenges that do not exist in any other application area of mobile robots must be overcome [10]:

- Complex environments: Offshore installations contain complex structures such as pipes, flanges, tanks, steel frames, stairways and many more. These structures can be very hard to detect by sensor systems applied typically in mobile robotics. The installed sensors on the mobile offshore robot must be able to distinguish relevant structures in the environment such as obstacles and free passages. The process equipment on offshore platforms is distributed among different levels, often including intermediate or mezzanine levels. only stairs or ladders are available to move from one level to the other. An appropriate means to move the robot from one level to another is required for a single robot to cover all levels.

- Floors: Offshore platforms on the contrary mainly consist of plain steel floor and gratings. There are many small holes, sharp edges, slopes and steps up to several centimeters in height.

- Explosive atmospheres.

- Corrosive environments: splash salty water, salty air and corrosive chemicals.

- Temperature: the ambient temperature on offshore platforms shows significant variations, depending on the platform's location. The mobile offshore robot must thus be operable in temperature ranges between $-30^{\circ} \mathrm{C}$ and $+50^{\circ} \mathrm{C}$.

- Humidity: The relative humidity up to $100 \%$ and condensing.

- Other conditions: possibly highly radiant heat from process equipment, heavy precipitation, storms, and direct sunlight.

\section{Current Robotics Technologies}

There are different kinds of robots in the oil \& gas industry. The remotely operated underwater vehicles are discusses first. Since there are many underwater pipeline problems, underwater pipeline repair robotic systems are developed to repair the pipelines. Mobile robot platforms for topside oil \& gas platform inspection and operations are investigated by academia and industry. Several systems are presented to understand their features and specifications.

\subsection{Remotely Operated Underwater Vehicles (ROVs)}

ROVs are unoccupied, highly maneuverable underwater robots operated by an operator aboard a surface vessel. They are linked to the ship by different cables that carry electrical signals back and forth between the operator and 
the ROV. Most are equipped with video cameras and lighting systems. Sometimes additional equipment is added to expand the vehicle's capabilities, including still cameras, manipulators or cutting arms, water samplers, and instruments that measure water clarity, light penetration, and temperature. ROVs were developed for industrial purposes, such as internal and external inspections of pipelines and the structural testing of offshore platforms; however, they are now used for many applications including scientific ones. They have proven extremely valuable in ocean exploration. They are also implemented for educational programs at aquaria.

ROVs vary in size from that of a bread box to a small truck. Deployment and recovery operations range from simply dropping the ROV from a small boat to complex deck operations using large winches for lifting and Aframes to take the ROV back onto the deck. In most cases, however, ROV operations are simpler and safer to conduct than many types of occupied-submersible or diving operations.

The disadvantages of using a ROV include: (a) the human presence is lost, making visual surveys and evaluations more difficult; (b) the lack of freedom from the surface due to the ROV's cabled connection to the ship. An ROV operator controls the vehicle from a system on board the ship using a joystick, a camera control, and a video monitor. The operator moves the vehicle and the camera to desired locations. The vehicle's depth, direction, and geographic position (latitude and longitude) are also recorded.

ROVs are often kept aboard vessels mounting submersible operations for several reasons. In the event that a submersible becomes entangled or otherwise incapacitated, an ROV can investigate the scene to help the operators make decisions. If appropriate, cutter blades can be deployed to the manipulator arm to free the sub. If a sub loses power and is not able to surface, the ROV's manipulator arm can grab onto the sub. The deck crew can then bring it to the surface.

ROVs also support exploration and science objectives. When the submersible cannot be used because of weather or maintenance problems, the ROV often can take its place. It can also be used to investigate questionable dive sites before a sub is deployed, limiting risk to the expensive subs and their pilots.

There are different kinds of ROV manipulators developed [11]-[13]. One example is the TITAN manipulator [11]. Constructed from titanium, the TITAN is uniquely capable of withstanding the industry's harsh and repetitive needs. Some features are:

- Seven degrees of freedom;

- Durable and reliable in harsh subsea environments;

- Large operating envelope;

- High lift-to-weight ratio;

- Depth rating from $4000 \mathrm{~m}$ to $7000 \mathrm{~m}$;

- Titanium construction;

- Hydraulic.

The master controller of the ROVs contains function keys for selecting configuration options and a display for viewing diagnostic and status information. The controller's advanced operational features include individual joint operation, position scaling (altering the ratio of master arm movement to manipulator arm movement), programmable routines, incremental gripper movement, individual joint diagnostics, and automatic error checking.

\subsection{Deep Water Pipeline Repair Robotic Systems}

Since there are many requests to repair deep water pipelines from the international oil and gas companies, StatOil [14] and Cheron [15] have developed pipeline repair robotic systems. The pipeline repair robots can work for water depths down to 1000 meters.

\subsection{SINTEF Topside Robotic System}

Because it is difficult to access subsea installations, normally-unmanned automated topside platforms may be an alternative through increased accessibility for large maintenance operations. Moreover, topside platforms may statistically recover up to 22 percent more of the oil or gas than a subsea alternative in a reservoir [7]. Due to these reasons, SINTEF worked with Norwegian energy producer StatoilHydro on a remotely operated offshore topside platform [8] [10] [16] in the robotic lab facility in Trondheim, Intelligent and reliable robotic and instrumentation systems has been developed to enable onshore operators to monitor and control all of the platform's processes.

Compared to their current manned counterparts, the normally-unmanned oil platforms offer potentially sig- 
nificantly lower commissioning and operation costs. Remote inspection and maintenance (I\&M) operations can be performed on offshore oil and gas platforms as an alternative to traditional offshore platforms. The platforms separate the work area accessible by human operators from a closed permanently unmanned area (PUA) serviced by robots. Some important scheduled I \& M operations inside the PUA can be performed by robots to replace human operators such as gauge readings, valve and lever operations and monitoring gas level, leakage, acoustic anomalies and surface conditions. Standard 6-axes robot manipulators (Kuka KR-16) are mounted on a mobile platform to automatically connect to custom-built tools and sensors such as vibration-measurement sensors, a valve-operating tool, and a lid operation tool. The operators will remain on land and are able to perform different tasks, reducing both risks and costs.

\subsection{Fraunhofer Inspection Robot}

The Fraunhofer inspection robot [17] was developed and tested by the Fraunhofer Institute of Manufacturing Engineering and Automation (IPA) for inspection. The robot uses specifically shaped objects such as pipes and poles as well as stripes of reflective tape applied to the environment for localization. It was tested continuously for 12 hours per day in hazardous locations and in tropical environment with $35+{ }^{\circ} \mathrm{C}$ ambient temperature, up to $90 \%$ rel. humidity, and direct sunlight. A laser scanner is used to perceive its environment. A six-axis lightweight arm installed on the robot carries a camera to perform visual inspections. The robot platform has various application-specific sensors, such as a stereo microphone as well as gas and fire sensors. Wireless LAN and Bluetooth are deployed to enable the robot to communicate with the central control PC and with a mobile operator control device.

The robot is capable of safe navigation in offshore environments, which enables the robot to autonomously record sensor data at key locations or continuously monitor sensor data along a predetermined path. A manual operator control device, such as an explosion-proof PDA, can be used to teach the robot to perform inspection tasks. The robot autonomously executes the inspection tasks after the inspection tasks have been taught. The sensor data are recorded and displayed at the central control PC. The operators in the central control room are able to supervise all relevant sensor data in real time. In addition, the robot can be teleoprated to assist the user by analyzing data of its environment sensors. The user drives the robot around the oil \& gas platform in order to get close to objects that need to be inspected or manipulated. A graphical user interface can display the objects detected by the robot through its sensors. The robot can then perform the planned tasks automatically such as executing movements or grasping operations to solve tasks, e.g. positioning its camera in front of a gauge or turning a hand wheel.

\subsection{CMU Inspection Robot}

The sensabot [18] was developed by Carnegie Mellon University, supported by Shell. Sensabot was designed for severe weather, pack ice, and temperatures that range from over $100^{\circ} \mathrm{F}\left(40^{\circ} \mathrm{C}\right)$ to below $-31^{\circ} \mathrm{F}\left(-35^{\circ} \mathrm{C}\right)$. On top of that, the robot should work in an environment with a corrosive, toxic, foul-smelling gas and explosive methane gas. Sensabot meets the International Electrotechnical Commission (IECEx) standards for electronic equipment and ANSI safety standards for guided industrial vehicles. The safety systems on Sensabot reduce the risks of environmental hazards and operating around personnel.

The mobile base of the Sensabot can deal with concrete, gravel, and steel gratings and tackles ramps, light snow, and slippery surfaces. It can go through human-sized walkways. It can access to multiple levels of a facility via ramps, elevators, and a cog rail systems.

There are different inspection sensors on Sensabot including (a) detectors to measure concentrations of gasses such as methane; (b) a powerful pan/tilt/zoom camera for remote operators to visually inspect pipes, valves and fittings for corrosion and other types of damage; (c) temperature sensors to help operators spot overheating equipment; (d) vibration sensors to enable operators to monitor the condition of pumps, motors, and bearings; (e) a microphone to help operators detect audible problems with machinery; (f) ladar and video to detect obstacles in its vicinity (such as equipment that lies in its path).

\section{Further Research Opportunities}

Because of the challenges and special requirements in the oil \& gas industry, there are many exciting research 
opportunities for robotics and automation. This section discusses the research areas in robot manipulators, mobile platforms, teleoperation and automated equipment.

\subsection{Manipulator}

Currently, pipe handling robots and ROV arms are two types of manipulators used in oil and gas. As people are looking for more automated oil rigs, specialized robot arms should be developed. The robot arms should be able to tolerate the harsh environments in the oil and gas areas. Besides that,

- The inspection robots should have a large workspace since the oil and gas equipment is typically much bigger than that in automotive manufacturing;

- The maintenance and/or repair robots should be able to handle big payload;

- The operation robots should have different tools;

- Light weight manipulator with big workspace for inspection;

- Heavy duty manipulator for operations.

\subsection{Mobile Platform}

ROVs are widely used in subsea operations to install equipment, perform maintenance and repair tasks etc. However there are many disadvantages for the current ROVs:

- Cables: the cables with the ROVs are easily tangled because they extend from the vessel to where the ROV is located. Since there are many objects in the sea, the cables could be tangled. Once ROV is tangled, the cables have to be cut.

- ROVs are not easy to operate. Even though there are some training systems to help the operators, it is a difficult task for the operators.

For the future research in the ROV, the opportunities could be:

- ROVs without using long cables from a vessel.

- Semi-autonomous ROVs. The ROVs can guide itself to avoid obstacles and locate the tasks.

- Autonomous ROVs. The ROVs are preprogrammed to perform given tasks before operations.

The ROVs with intelligence can make decisions and perform given tasks. Besides ROVs, mobile platforms will have many applications in oil and gas:

- Topside inspection and monitoring;

- Topside maintenance and repair;

- Topside operations.

The future research for the mobile platforms could be:

- Mobile platforms should be able to tolerate harsh environments;

- Mobile platforms should be able to anchor themselves automatically;

- Teleoperated mobile platforms could be more feasible in a short term.

Due to the development of unmanned platforms on the surface and teleoperated seafloor drilling rigs, mobile manipulators will parallel deep sea ROVs in maintaining platforms, where it is reasonable to assume the industry will see many more unmanned platform projects in the very near future.

\subsection{Teleoperation}

Teleoperation will still be the enabling technology in a short term because the oil \& gas industry hesitates about using fully automated robotic system on the oil and gas operations. Different teleoperation technologies should be developed for different applications, for example, mobile platforms on the rig and subsea mobile platforms.

\subsection{Subsea Robots}

Half of the world's remaining oil reserves lie beneath the oceans' floors. Getting to them presents distinct engineering challenges, ranging from weather- and ice-related issues to the environmental and human safety risks.

Automated, robot-operated, unmanned drilling rig that sits on the bottom of the sea would resolve the problems related to weather-tossed seas, darkness, floating icebergs, and pack ice. Instead of building an offshore surface platform to drill for oil and gas at the seabed, up to 3000 meters below, exploration companies can use the Seabed Rig system to drill on the sea floor using robotic systems, with only a support vessel at the surface. 
The Seabed Rig also eliminates the potential for human error, is safer, and costs less than conventional drilling platforms.

There are many opportunities for robotic system in seabed operations. Besides drilling, other possibilities are:

- Automated seabed maintenance and repair system;

- Automated seabed inspection system;

- ROVs.

\subsection{Automated Equipment}

Because the oil and gas industry does not require highly flexible systems, hard automation could be better solutions for the industry. For example, seabed drilling system is more like a hard automation system, instead of robotic system. Furthermore, Robotic drilling systems, such as the one developed by Seabed Rig, would appear to be extremely useful systems for simplifying the drilling process and ensuring that its accuracy is to the utmost degree. Most drilling processes are extremely labor intensive and require continual human supervision, which could be easily remedied by robotic systems.

\subsection{Related Research Topics}

Since an offshore platform is quite complex, there are many wires used to transmit signals or transfer power. Data communication is a critical issue. Some potential research areas are:

- Sensor network;

- Wireless data communication;

- RFID applications;

- Data management.

Since the paper focuses on exploring robotic applications in the oil \& gas industry, the above research areas will be discussed in our future research.

\section{Conclusions}

There is little doubt that the oil \& gas companies would benefit greatly from the use of more intelligent technologies, not only increasing their efficiency, but also cutting down on human risk factors that are ever present in rig environments.

There is a clear incentive for oil and gas companies to automate their oil and gas facilities, starting with isolated operations, such as pipe handling and assembly for drilling and tasks related to rig operations. These examples represent high-risk operations for humans and therefore provide opportunities to improve health, safety and environmental (HSE) performance.

In addition to productivity and efficiency gains, robots used for high-risk tasks will also lead to improvements in HSE performance. Such tasks are not necessarily always predictable and represent unusual robot activities. The robot will therefore require features that extend the "eyes, ears, and hands" of the human decision makers as they carry out inspections and maintenance operations on the process infrastructure.

Reduced commissioning and operation costs, together with improved Environmental, Health and Safety (EHS) are some of the potential benefits of having normally unmanned topside oil platforms. However, such oil \& gas platforms require advanced methods and tools for remote control and monitoring of inspection and maintenance operations.

In this paper, a brief introduction to the oil \& gas industry processes is performed. The challenges and requirements for the robotics and automation equipment are explored. Future research opportunities including robot manipulator, mobile platform, teleoperation, and subsea robotics are discussed. Overall, we postulate that there are many opportunities in the oil and gas industry and some research is currently in progress to develop robotic and automation applications. Therefore, it is an optimal time to develop robotic and automation systems that can satisfy the oil \& gas requirements.

\section{Acknowledgements}

The authors would like to thank ABB Corporate Research Center, ABB Inc. Windsor, Connecticut, for supporting the study. 


\section{References}

[1] World Nuclear Association (2012) Uranium, Electricity and Climate Change. http://www.world-nuclear.org/info/Energy-and-Environment/Uranium,-Electricity-and-Climate-Change/

[2] Upstream (Petroleum Industry). (2014). http://en.wikipedia.org/wiki/Upstream_(petroleum_industry

[3] Upstream Oil and Gas. (2014). http://www.upstreamoilandgas.com/

[4] Gary, J.H. and Handwerk, G.E. (1984) Petroleum Refining Technology and Economics. 2nd Edition, Marcel Dekker, Inc., New York.

[5] GE Energy. (2011). http://www.ge-energy.com/solutions/index.jsp\#tabs-industries

[6] US Environmental Protection Agency (2000) US EPA Office of Compliance Sector Notebook Project: Profile of the Oil and Gas Extraction Industry. US Environmental Protection Agency, Washington DC.

[7] Meeting the Challenges of Today’s Oil and Gas Exploration and Production Industry. http://www-935.ibm.com/services/us/gbs/bus/pdf/g510-3882-meeting-challenges-oil-gas-exploration.pdf

[8] Transeth, A.A., Skotheim, Ø., Schumann-Olsen, H., Johansen, G., Thielemann, J. and Kyrkjebø, E. (2011) A Robotic Concept for Remote Maintenance Operations: A Robust 3D Object Detection and Pose Estimation Method and a Novel Robot Tool. The International Conference on Intelligent Robots and Systems, Taipei, 18-22 October 2010, 5099-5106.

[9] The Robotized Field Operator. http://www.isa.org/InTechTemplate.cfm?template=/ContentManagement/ContentDisplay.cfm\&ContentID=79568

[10] Kyrkjebø, E., Liljebäck, P. and Transeth, A.A. (2009) A Robotic Concept for Remote Inspection and Maintenance on Oil Platforms. Proceedings of ASME 28th International Conference on Ocean, Offshore and Arctic Engineering (OMAE 2009), Hawaii, 31 May-5 June 2009, 667-674.

[11] Schilling Robotics. (2014). http://www.schilling.com/products/manipulators/Pages/default.aspx

[12] Atlas Hybrid Manipulator. (2014). http://www.oceaneering.com/rovs/rov-technologies/atlas-hybrid-manipulator/

[13] ECA Robotics. (2014). http://www.eca-robotics.com/en/control-command-security/robotics/2.htm

[14] Statoil Developing Deepwater Pipeline Repair Robot. (2014). http://www.offshore-mag.com/articles/2007/03/statoil-developing-deepwater-pipeline-repair-robot.html

[15] Chevron's Deepwater Pipeline Repair System Revealed. (2013). http://oil-geneva.blogspot.com/2013/02/chevrons-deepwater-pipeline-repair.html

[16] Robots Taking over the Job on Offshore Drilling Platforms. (2014). http://www.sciencedaily.com/releases/2007/12/071221230852.htm

[17] MIMROex. Fraunhofer Institute of Manufacturing Engineering and Automation. http://www.ipa.fraunhofer.de/MIMROex.2297.0.html?\&L=2

[18] SensaBot Inspection Robot. Robotics Institute, Carnegie Mellon University. http://www.ri.cmu.edu/research_project_detail.html?project_id=752\&menu_id=261 
Scientific Research Publishing (SCIRP) is one of the largest Open Access journal publishers. It is currently publishing more than 200 open access, online, peer-reviewed journals covering a wide range of academic disciplines. SCIRP serves the worldwide academic communities and contributes to the progress and application of science with its publication.

Other selected journals from SCIRP are listed as below. Submit your manuscript to us via either submit@scirp.org or Online Submission Portal.
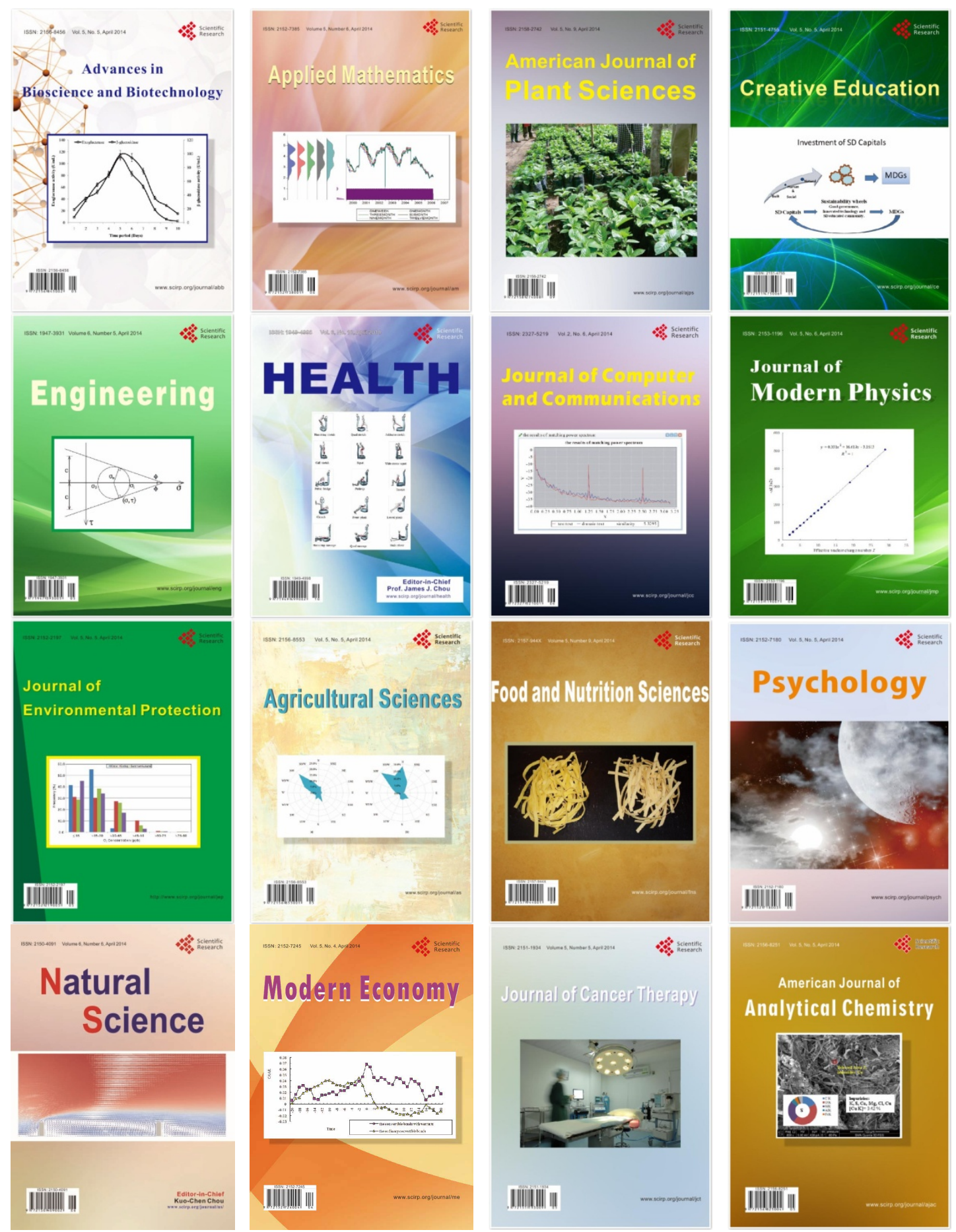